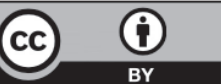

https://creativecommons.org/licenses/by/4.0/

\title{
DISEÑO DE MÓDULOS INTERACTIVOS PARA TRATAR EL TRASTORNO POR DÉFICIT DE ATENCIÓN CON HIPERACTIVIDAD - TDAH*
}

\author{
Interactive modules design to treat attention-deficit hyperactivity disorder
}

EVELYN GARNICA ESTRADA**, BREINER FRANCISCO QUIROGA LÓPEZ***, PEDRO MIRANDA CASTRO****, ÁNGELA PATRICIA MEDINA LEÓN*****

Recibido: 25 de abril de 2016. Aceptado: 22 de junio de 2016

DOI: http://dx.doi.org/10.21017/rimci.2016.v3.n6.a14

\begin{abstract}
Resumen
El presente artículo describe la solución planteada a través de software y módulos físicos para el tratamiento del trastorno por déficit de atención con hiperactividad - TDAH; proyecto propuesto con el fin de ofrecer otra alternativa de terapia de bajo costo con el fin de potenciar habilidades en los niños que padecen dicha condición.

Palabras clave: juego, interfaz, terapia, software.
\end{abstract}

\section{Abstract}

This article describes the solution proposed through physical modules and software for treating attention deficit hyperactivity disorder - ADHD; proposed project in order to provide alternative low-cost therapy in order to enhance skills in children with this condition.

Keywords: game, interface, therapy, software.

\section{INTRODUCCIÓN}

La implementación realizada en el marco del proyecto de investigación desarrollado en la Corporación Universitaria Republicana, se centra básicamente en el desarrollo de módulos interactivos para apoyar a padres y profesionales en el tratamiento multimodal del déficit de atención e hiperactividad en niños en edades entre 4-7 años.

El proyecto consta de dos partes, un conjunto de módulos de software de juegos interactivos y un conjunto de módulos físicos que sirven como controles de entrada para manipular de forma natural la parte virtual del juego.

A pesar de que se trata de un juego con componentes virtuales, el niño siempre esta inmerso en la realidad física, característica fundamental que convierte el juego en una terapia y el software se convierte en instrumento de apoyo para la misma. Se puede definir este proyecto como la integración de mundos físicos y virtuales para que el niño experimente ambos ambientes, con alta prioridad en lo físico.

* Resultado del trabajo de semillero en el marco del proyecto: «Desarrollo de Juegos interactivos para niños hiperactivos» del grupo de investigación GIDIS (Grupo de Investigación y Desarrollo de Ingeniería de Sistemas) de la Corporación Universitaria Republicana.

** Ingeniera de Diseño y Automatización Electrónica de la Universidad de la Salle. Especialista en Planeación, Desarrollo y Administración de la Investigación de la Universidad Manuela Beltrán. Magíster en Dirección de Proyectos de la Universidad Viña del Mar. Actualmente cursando Doctorado en Educación en la Universidad de Baja California. Docente Investigadora de la facultad de Ingeniería de la Corporación Universitaria Republicana. Correo electrónico: egarnicae@urepublicana.edu.co

*** Estudiante de Ingeniería de sistemas de la Corporación Universitaria Republicana. Correo electrónico: esclesais-provais1@hotmail.com

****Estudiante de Ingeniería de sistemas de la Corporación Universitaria Republicana. Correo electrónico: pedromiranda7777@gmail.com

*****Estudiante de Ingeniería de sistemas de la Corporación Universitaria Republicana. Correo electrónico: patito_8080@hotmail.com 
Para planear el diseño de los módulos se inicio una investigación acerca de los juegos que se utilizan en el tratamiento psicoeducativo de los niños con TDAH (Trastorno por déficit de atención con hiperactividad) para tomarlos como base de los prototipos para trabajar competencias académicas que debería tener un niño de acuerdo a su edad y poderlas aplicar sobre los módulos.

\section{Planteamiento del Problema}

El trastorno de déficit de atención con hiperactividad que se caracteriza por impulsividad e inatención prevalece en la población con un porcentaje entre el 1 y el 20 por ciento [1], además esta condición tiene influencia en el comportamiento social de la persona, es decir, que las personas que sufren las condiciones de hiperactividad e impulsividad con comportamiento destructivo tiene más posibilidades de ser detenidos cuando son adultos, por tanto, la prevalencia del TDAH en las personas lleva a la persona desde la adolescencia a consumir sustancias psicoactivas.

Los niños que padecen esta condición y no son tratados tienen más tendencia a tener fracaso escolar, a consumir sustancia psicoactivas, sufren accidentes frecuentes, conducta antisocial y posible conducta delictiva [2]. Por otra parte, existe una prevalencia en las personas afectadas no tratadas que conlleva en adolescentes a conducta agresiva, delincuencia, consumo de alcohol y drogas, sistemas depresivos emocionales, embarazos no deseados, problemas legales, y en adultos esto conlleva a problemas familiares y en sus relaciones interpersonales como en el matrimonio, dificultad en el trabajo, en el manejo del dinero, fracasó en su carrera profesional, problemas con proyectos a largo plazo, entre otros.

Para algunas familias de los niños que padecen la condición, se considera una situación secundaria y atribuida a una inadecuada disciplina, padres muy flexibles o gracias a los sistemas muy inapropiados en escuelas y colegios, pero hoy en día esta claro en los estudios realizados que esa condición de TDAH tiene unas connotaciones más amplias y debido a la falta de atención, lo niños son diagnosticados inadecuadamente y en muchas ocasiones nunca se atiende los síntomas a estas personas [3].
Estudios recientes han indicado a nivel mundial que se tiene un 8 a $12 \%$ en niños y adolescentes, y de 1.2 a $7.3 \%$ en adultos que padecen el trastorno, y como se ha visto anteriormente esta condición implica dificultades sociales, psicológicas (baja autoestima), de aprendizaje, complejos de fracaso académico o profesional, consumo de sustancias psicoactivas y delincuencia tanto en la adolescencia como en la edad adulta. La calidad de las relaciones de estas personas se deteriora no sólo con sus compañeros y vecinos sino con su familia directa como padres y hermanos. Debido a su condición de inatención a las normas sociales y a su conducta hostil conlleva estrés por sentimientos de incompetencias y situaciones de discordia [4].

El trabajo «Trastorno por Déficit de Atención e Hiperactividad- Guía para Padres» realizado por la Fundación de Neuropsicología Clínica, en su apartado « $i$ El trastorno se circunscribe a la infancia? «, cita que las investigaciones actualmente establecen que de 100 niños menores de 12 años que recibieron diagnóstico de TDAH cuando se volvieron a evaluar como adolescentes un $70 \%$ de ellos todavía eran diagnosticados con TDAH y en la edad adulta esta cifra no baja del 50 \% [5].

Analizando todas las citas referenciadas anteriormente indican que el trastorno de déficit de atención e hiperactividad es un problema mundial y existe la necesidad que tanto padres, familia, profesores, profesionales en el tema y gobierno deben comprometerse para tratar urgente y adecuadamente a los niños y/o personas que sufren esta condición para mejorar sus condiciones de vida y poder formar personas útiles a la sociedad ya que se ha visto que algunos de estos síntomas persisten en las personas aun en la edad adulta dificultando la vida cotidiana no sólo del individuo en particular, sino también para su familia y la sociedad en general.

En las investigaciones consultadas, se encuentra que el tratamiento de esta condición es multimodal [6], se requiere un tratamiento psicológico, conductual psicoeducativo o pedagógico y el uso de fármacos, además, que requiere no solo el compromiso del niño, sino el de toda su familia ya que estos últimos, representan una parte fundamental en la rehabilitación del individuo, pero, los profesionales y los padres prefieren desde el principio el uso de medicamentos para tratar el problema del

Rev. Ingeniería, Matemáticas y Ciencias de la Información Vol. 3 / Núm. 6 / julio - diciembre de 2016; pág. 49-57 
TDAH en sus hijos debido a que es una estrategia más cómoda en tiempo y dinero, que un proceso psicopedagógico para enseñar al niño las destrezas y competencias académicas que necesita de acuerdo a su edad y que no las ha alcanzado debido a su condición, además, de las destrezas sociales, de autocontrol y de motivación personal para la realización y finalización de tareas cotidianas.

No se puede afirmar que no se requiera medicamentos para tratar el TDAH y tampoco que si se necesite, pero lo que sí es válido es que la terapia psicopedagógica y conductual es fundamental en el tratamiento del TDAH y tomando como referencia este tipo de tratamiento, se necesitaría diseñar un sistema de módulos interactivos que brinde a los profesionales, a los padres y la familia en general y a los profesores, unas herramientas lúdicas, psicoeducativas que enfaticen sobre los problemas que se presentan en los niños con esta condición, como lo son, la atención, la impulsividad destructiva, la conducta y el aprendizaje.

Bajo este contexto y en el marco de la elaboración del trabajo de grado se presento el siguiente planteamiento ¿De qué forma se podrían diseñar herramientas psicopedagógicas que apoyen a profesionales, padres y familia en general de niños que presentan TDAH, a reforzar el tratamiento terapéutico que facilite la rehabilitación inmediata de ellos sin el uso de medicamentos trayendo consigo beneficios económicos y un gran impacto positivo tanto al propio individuo como a su familia y la sociedad en general?

\section{OBJETIVO GENERAL}

Diseñar un sistema interactivo terapéutico para niños con déficit de atención e hiperactividad (TDAH) en edades entre los 4-7 años mediante el empleo de herramientas de hardware y software que facilite en ellos la concentración, el desarrollo y finalización de tareas, el control de la impulsividad y el comportamiento destructivo, y refuerzo del aprendizaje escolar.

\section{A. Objetivos específicos}

1. Identificar los juegos interactivos que utilizan los profesionales en el tratamiento psicopedagógico en niños con TDAH.
2. Identificar las competencias académicas que se les debe impartir a los niños según su rango de edad.

3. Diseñar el sistema de módulos interactivos tanto el software como el hardware que lo controla para el tratamiento psicopedagógico.

4. Realizar pruebas en niños con TDAH para verificar su desempeño y funcionalidad en la rehabilitación de su condición.

\section{IDENTIFICACIÓN DE SOLUCIONES EXISTENTES PARA EL TRATAMIENTO PSICOPEDAGÓGICO EN NIÑOS CON TDAH}

Existe una propuesta teórica que enfatiza el uso de los videojuegos para el tratamiento del TDAH, donde se advierte que no cualquier juego podría servir para tratar esta condición y depende de su correcta utilización para obtener efectivos resultados, así mismo se muestra algunas pautas del perfil del juego como lo son: cooperativo, social, metas definidas, consecución de logros y recompensas, de niveles, etc. En dicha investigación se evidencia mejoras en el autocontrol, motivación en la ejecución de tareas escolares y se ha referenciado una disminución del uso de fármacos en los niños tras el tratamiento con video juegos [7].

Otros estudios realizados en niños con TDAH, emplean las tecnologías de la computación para el tratamiento de la hiperactividad; denominada «Neurofeedback», que estudia la actividad cerebral y los impulsos nerviosos dependiendo de lo que hacen las personas. Este juego consiste en un aplicativo de software que se controla a través de la mente, el niño lleva un brazalete en la mano que va recogiendo la actividad neuronal y los elementos se activan de acuerdo a la atención que tiene el niño en el juego, esto fomenta el aprendizaje, la motivación y el autocontrol. Este juego se ha aplicado con éxito en niños, en pocas sesiones se ha conseguido que los niños muestren cambios reales tanto en la atención como en la conducta [8].

Así como el Neurofeedback, existen otras tecnologías asociadas como el Atention Training Systems usado para enfatizar la atención en los niños, se debe concentrar en el juego para poder 
mover los objetos en el juego, y el Biofeedback, que se centra en el auto control y la relajación ya que el jugador debe relajarse lo máximo posible para que el juego responda [8].

En estudios realizados por el Instituto para la Salud del comportamiento en Commack, Nueva York, indican estadísticas positivas en pruebas realizadas, estas pruebas arrojaron una reducción del $80 \%$ en la hiperactividad y un $82,2 \%$ en la mejoría de la atención; también otros investigadores del Tufts Medical Center, en sus estudios reportan aspectos positivos frente al tratamiento del TDAH [9].

Por otro lado, la Empresa «Starlab», ha desarrollado una aplicación de software con la que se mide la actividad cerebral de los niños y se traslada a un videojuego. El objetivo es tratar el déficit de atención y la impulsividad, tras detectar las emociones para transformarlas en comandos que pueden controlar el videojuego [10].

MeMotiva, es un programa diseñado para el TDAH, por instituto de Pedagogía y Educación Especial de Suecia, se trata de una aplicación diseñada para aumentar la capacidad de la memoria operativa en niños y adultos con este trastorno. El programa consiste en una serie de juegos viso-espaciales y viso-auditivos para desarrollar la memoria operativa y la atención [11]. Al principio los niños deben hacer su perfil con un test de nivel para que las actividades estén adaptadas a los alumnos. Además, los ejercicios van progresando poco a poco y cada cierto número de ejercicios, baja un poco nivel para que los alumnos no se frustren.

En una investigación de Semrud-Clikeman, Nielsen, Clinton, Sylvester, Parle y Connor en el año 2005, se examinó los efectos del entrenamiento de la atención en niños con TDAH con el programa MeMotiva y los resultados del estudio que tardaron 18 semanas, concluyen que los niños puestos en el grupo de entrenamiento mejoraron significativamente en un test de atención viso-auditivo frente los niños que fueron colocados en el grupo de control. La página oficial de la aplicación Rehasoft muestra las estadísticas positivas del estudio realizado [12].

Se encontraron estudios más avanzados como por ejemplo una aplicación con una serie de videojuegos de alta tecnología creados por la
NASA y la Fuerza Aérea de EE.UU para entrenar y mejorar la atención y concentración, mejora las habilidades cognitivas y modifica la conducta. Esta aplicación funciona con un sistema llamado Bodywave, basado en un brazalete que registra las señales cerebrales de atención, y luego las transmite por Bluetooth al software, que registra los datos continuamente, los cuales se reflejan en el juego de manera que el usuario puede ver como evoluciona su atención de una forma motivadora. Además la atención se convierte en la energía del juego, de forma que si el niño no atiende el juego no funciona. Luego de que el niño utiliza el juego, la plataforma se sigue utilizando con el niño para que el realice sus tareas cotidianas midiendo el grado de atención que le coloca a estas y evaluar la efectividad de la aplicación en el tratamiento psicopedagógico [13, 14].

Para los especialistas, el uso de los videojuegos en el tratamiento de los niños con TDAH, se vuelve una herramienta sencilla y una buena alternativa ante otros tratamientos como el farmacológico que puede tener efectos negativos secundarios sobre los niños tratados.

\section{IDENTIFICACIÓN DE DINÁMICAS Y COMPETENCIAS}

En el proceso de identificación de dinámicas se encontró el tratamiento terapéutico y los ejercicios asociados, así como las competencias en los rangos de edad establecidos, lo que permitió diseñar los módulos propuestos.

\section{A. Tratamiento terapéutico}

El tratamiento terapéutico se puede realizar en el hogar basado en de juegos caseros que pueden resultar útiles a profesionales, profesores y padres en el tratamiento terapéutico de los niños con TDAH [15]. Estos juegos con mejoras a través de tecnología podrían ser más eficientes no solo para el niño sino para sus padres, además de apoyar a la rehabilitación de los niños con esta condición; a continuación se muestran una lista de estos ejercicios:

1. Ejercicios para la atención

a. Completar rompecabezas de letras y de figuras geométricas de dificultad creciente.

Rev. Ingeniería, Matemáticas y Ciencias de la Información Vol. 3 / Núm. 6 / julio - diciembre de 2016; pág. 49-57 
b. Completar secuencias de trazados.

c. Un buen juego para potenciar la atención en niños hiperactivos es resolver laberintos, sopas de letras y crucigramas e ir añadiéndoles dificultad.

2. Juegos para practicar la memoria auditiva y visual

a. Juego del eco: el guía dice una palabra y los demás deben hacer el eco de la misma las veces que se acuerde previamente.

b. Juego del mensajero: el guía dice a otro jugador una frase relacionada con alguna actividad a realizar y éste debe repetirla posteriormente a todo el grupo para que la lleven a cabo.

c. Juego de las cadenas de palabras: el guía inicia la cadena diciendo una palabra ( $\mathrm{p}$. ej. «Juanito»), el siguiente jugador debe decir la misma palabra más otra que añada significado a la primera (p. ej. «está»)... pierde el jugador que no es capaz de añadir otra palabra más.

3. Ejercicios para practicar la coordinación viso motriz

a. Buscar el detalle: En este ejercicio se le muestra al niño diferentes dibujos de objetos, fotografías e imágenes en un periodo breve de tiempo. Después se le pregunta qué detalles recuerda y se le pide que dibuje la imagen cuidando la disposición, el color y el tamaño, cuantos más detalles recuerde más elogios recibirá. Puede realizarse también con personajes.

b. A clasificar: Consiste en clasificar objetos distintos según un criterio dado o una característica común. Se podrá ampliar aumentando el número de características comunes y utilizando materiales cotidianos.

c. El laberinto.

4. Ejercicios para practicar la relajación y el control de la impulsividad a. El globo. Sirve para practicar la respiración diafragmática. Inspirar muy despacio permitiendo que el aire entre por los pulmones y llegue al abdomen. Este último se va hinchando como si fuera un globo y después permitiendo que se escape el aire y desinflándose poquito a poco hasta quedar vacío.

b. La tortuga en su caparazón. Este ejercicio permitirá practicar la relajación muscular progresiva. Nos tumbamos boca abajo y nos convertimos en una tortuga que decide replegarse en su caparazón escondiendo su cabeza y sus patas. Ahora los músculos del cuello, brazos y piernas están en tensión. Pronto vienen los rayos del sol a despertarnos, ahora vamos asomando muy despacito la cabeza y estirando las extremidades hasta que queden relajadas. Podemos completarlo con «la historia de la Gran Tortuga Sabia» (basada en «la tortuga de M.R. Schneider y A. Robín»).

\section{B. Competencias académicas a fortalecer}

A continuación se presentara algunas de las competencias académicas para niños de 4 a 6 años que se deben tener en cuenta para utilizarlo en los módulos y asi, mientras ellos se van rehabilitando jugando también van reforzando sus capacidades académicas que no hayan podido obtener a causa de padecer esta enfermedad.

\section{Matemáticas}

Los números hasta el 100, aprender a contar, asociación, seguir la secuencia, trazos, laberinto, conceptos, figuras geométricas, conceptos (pequeño, mediano, grande, arriba, en medio, abajo, en frente, atrás, adentro, afuera). Para 5 años en adelante: conceptos (alto, bajo, largo, corto, pertenencia, no pertenencia, junto, separado, muchos pocos, izquierda, derecha, cerca, lejos, encima, debajo, grueso, delgado, encima, debajo, adelante, detrás, arriba, abajo, dentro, fuera), conjuntos, mas que, menos que, igual, seriaciones, mitades, subconjuntos, cuerpos geométricos. 


\section{Lengua}

Vocales, abecedario, asociación, laberinto, trazos, secuencia, conceptos. Para 5 años en adelante: lectoescritura.

\section{Ciencia}

Asociación de animales, plantas, frutas, conceptos de cosas, trazos, objetos, elementos. Para 5 años en adelante: el nombre de los dedos, ciclo productivos de los alimentos.

\section{Sociales}

Himnos nacionales, bandera, escudo, datos personales del niño.

\section{Colores}

Asociación, pintado. Clasificación, selección.

Tanto los ejercicios y juegos que utilizan los profesionales en el área para el tratamiento del TDAH en niños como las materias curriculares que uno ve en la escuela en las edades entre 4-7 años mencionadas anteriormente, van a ser utilizadas como guias de apoyo e inspiración, ya que a través de este tipo de juegos se intentara reproducir módulos y funcionales que puedan servir como un control de aplicaciones interactivas que se desarrollaran en conjunto con ellos. Tanto los dispositivos físicos como las aplicaciones interactivas de software se utilizaran como una herramienta de apoyo terapéutico en el tratamiento del TDAH en los niños de las edades antes mencionadas.

\section{C. Áreas a trabajar}

A continuación se plantearan todas las áreas que se tendrán en cuenta para el desarrollo de los módulos interactivos que favorece el aprendizaje y la ejercitación para el desarrollo de un niño como lo son:

1. Área Sensorial: sentidos y percepción.

2. Área Motriz: motricidad fina, motricidad gruesa y propiocepción.

3. Área Cognitivo: memoria, atención, cognición, procesamiento lógico.
4. Área comunicativa: lenguaje, expresión, interacción, diálogos, rituales.

5. Área afectiva: superación de miedos, angustias, fobias.

6. Área social: roles, competencia, resuelve conflictos.

\section{SOLUCión PROPUESTA}

En el mercado existen muchas herramientas de tipo psicopedagógicas que se basa en juegos interactivos de computador para el tratamiento del TDAH en niños, adolescentes y adultos, pero estos sistemas simulan toda la realidad a nivel virtual, dejando solo el teclado, el mouse o controles básicos de juego que no le dan al usuario más interacción con el juego que unas simples pulsadas de algunos botones.

Existen otras plataformas de juego como el Xbox de Microsoft o los sistemas de realidad virtual que permiten que uno mismo sea el control en el juego, lo que llevo a la idea de generar controles de juegos reales inmersos en juegos virtuales, y es aquí donde no se utiliza el mouse ni el teclado si no los modulos físicos propuestos.

La idea es entregarle al niño el total control del juego, dándole la interacción que se requiere con su entorno ya que esto es muy fundamental en el desarrollo neuropsicológico de cada persona, ya que como indican diversos estudios, el juego le debe proporcionar al niño la interacción con su medio ambiente y social, ya que a través de la interacción con su entorno, el niño va adquiriendo todas las habilidades y destrezas tanto psicológicas, como académicas, motrices, cognitivas y sociales que le permiten crecer como una persona feliz y útil para el mismo y para la sociedad en la que vive.

Herramientas tecnológicas como MAKEYMAKEY inventados por Silver y Rosenbaum [16], abren la posibilidad de convertir casi todos los objetos del entorno en un sistema de control para el juego, ya que simula el teclado y mouse, siendo una alternativa de bajo costo, abierta y flexible para generar una buena plataforma interactiva de software, que permite desarrollar una solución para rehabilitar a niños con TDAH mediante el juego. 
Se diseñaron 5 módulos físicos:

\section{a. Laberinto Topológico}

Es un módulo diseñado para niños desde 5 a 7 años de edad, donde se potencian las diferentes habilidades: Coordinación visomotriz, coordinación del espacio topológico, concentración y control de la impulsividad.

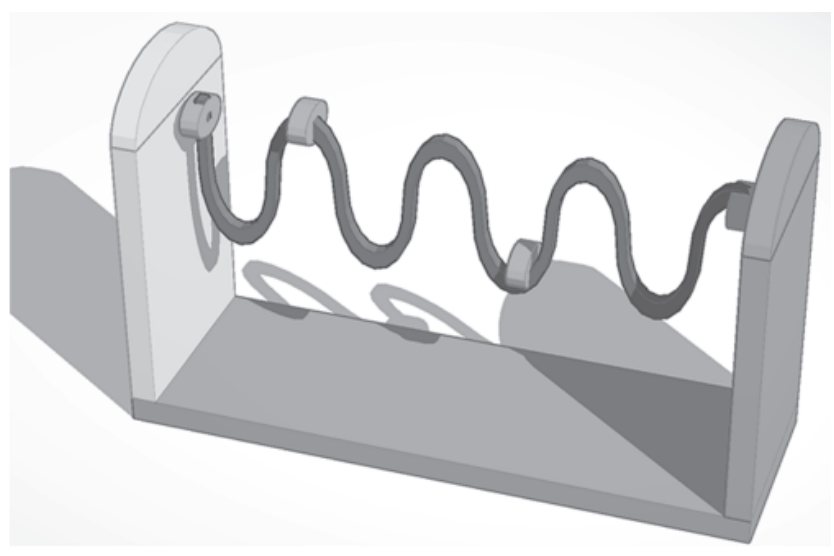

Fig. 1. Diseño módulo físico del Laberinto Topológico.

\section{b. Rompecabezas}

Es un módulo diseñado para niños desde 4 a 6 años de edad, donde se trabaja la ejercitación de la atención, coordinación visomotriz y ejercitación de la memoria visual.

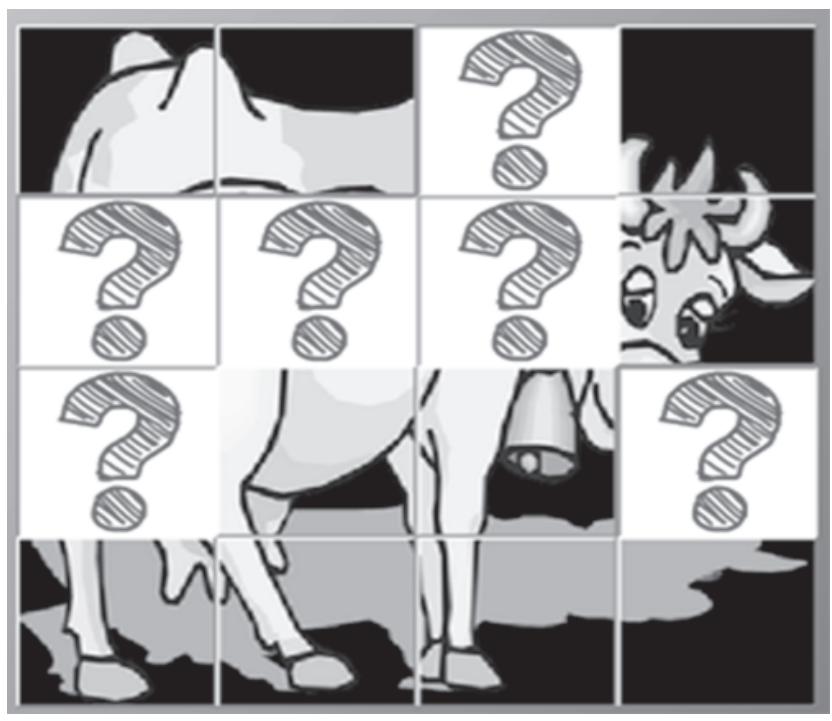

Fig. 2. Diseño módulo físico del Rompecabezas.

\section{c. Orientación Espacial}

Es un módulo diseñado para niños desde 4 a 7 años de edad, diseñado para trabajar la orientación espacial y la memoria visual.

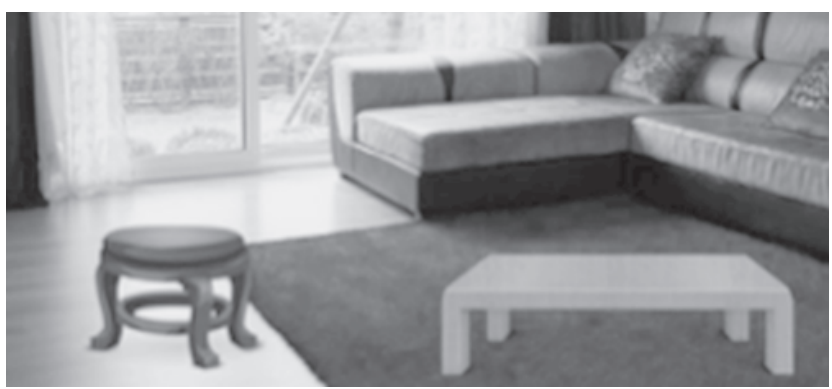

Fig. 3. Diseño módulo de orientación espacial - ubicación de objetos.

\section{d. Clasificación de objetos}

Es un módulo diseñado para niños desde 4 a 6 años de edad, ideal para trabajar la coordinación visomotriz y la atención a las características.

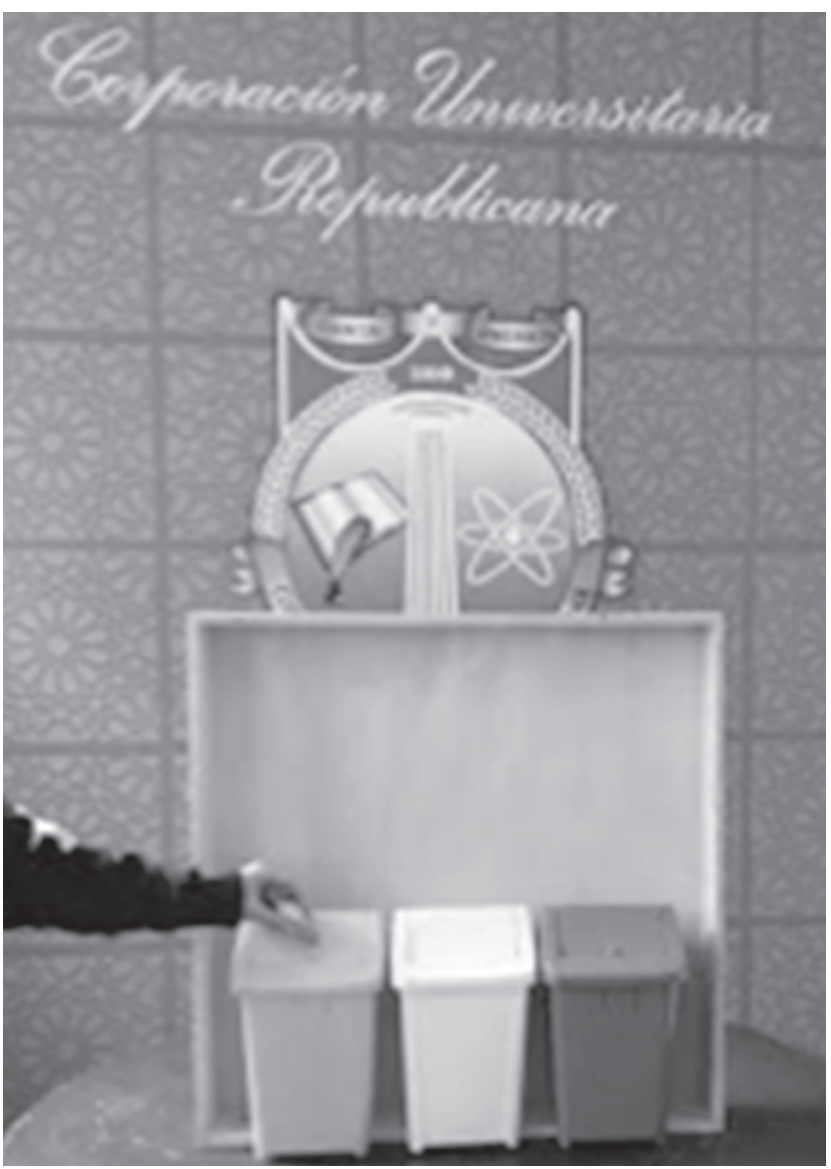

Fig. 4. Diseño módulo de clasificación de objetos - Reciclaje. 


\section{e. Secuencia de Figuras Geométricas}

Es un módulo diseñado para niños desde 5 a 6 años de edad, donde se trabaja la atención de los niños, la memoria visual, la coordinación visomotriz, la topología de las figuras y las características de las figuras geométricas.

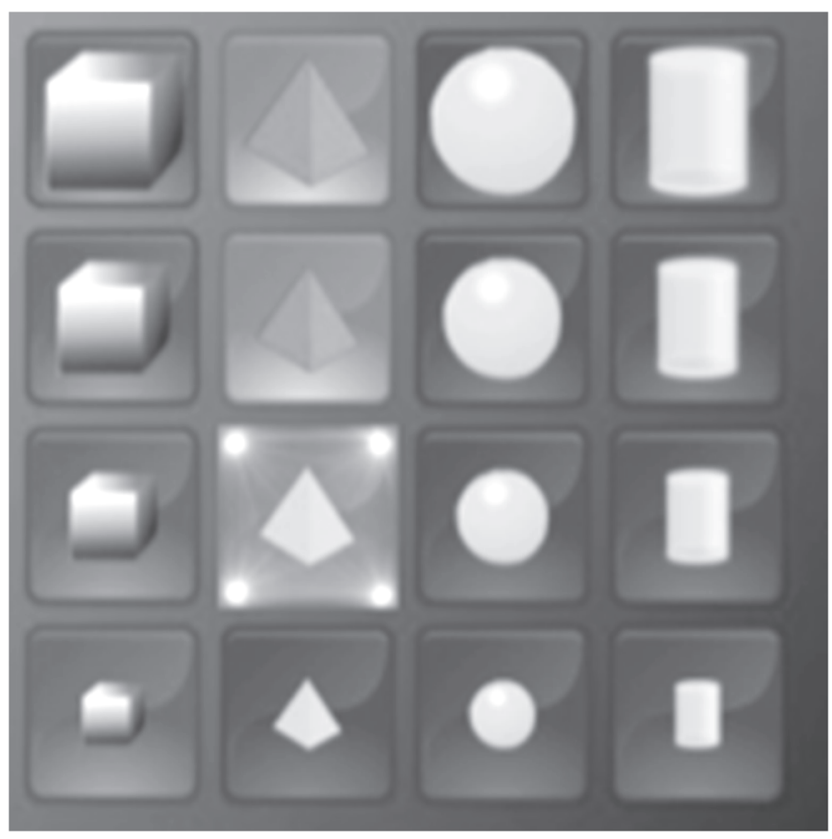

Fig. 5. Diseño módulo de Secuencia de Figuras geométricas.

Con base a los cinco diseños planteados anteriormente, la construcción de cada estructura física ha sido trabajada con materiales convencionales, como madera, plástico, papel, cartón y demas materiales que sean de fácil acceso y de uso cotidiano, con el fin de que puedan ser reproducidos por padres, familiares y profesionales pedagogos en casa y colegios. Según sea el caso, la idea original es producir los módulos y distribuirlos a padres, profesores y profesionales por un valor sostenible y asequible al público interesado.

\section{CONCLUSIÓN}

El proyecto se encuentra en fase de desarrollo, donde se espera terminar los cinco módulos físicos y realizar las interfaces correspondientes de software. Una vez terminada la fase de implementación del proyecto, se realizarán pruebas con niños que padecen la condición, para validar las interfaces del juego y el funcionamiento de todo el sistema.

El proyecto es escalable lo que permite generar otro tipo de módulos para potenciar más las habilidades y competencias de los niños.

Inicialmente se planteó cinco módulos pero cada uno de ellos conlleva a niveles de complejidad para garantizar un progreso en la ejecución de las terapias.

Como se ha dicho a lo largo del presente artículo, el TDAH o Trastorno de Déficit Atencional e Hiperactividad es una condición que perjudica no solo a los niños que la padecen, sino también a sus familiares, profesores y amistades, por esta razón se propuso una solución de software y hardware como instrumento tecnológico para abordar las terapias de una forma más fácil y divertida; se espera que al utilizar los juegos interactivos de forma adecuada podría ayudar a la rehabilitación terapéutica de ellos sin necesidad de utilizar tratamiento con fármacos como siempre se ha considerado.

En el transcurso del proyecto, se trabajará en otras publicaciones detallando el desarrollo del software, la integración con el hardware y las pruebas.

\section{Agradecimiento}

Este proyecto ha sido apoyado por el Grupo de Investigación y Desarrollo GIDIS de la Corporación Universitaria Republicana en el marco de semillero de investigación.

\section{REFERENCIAS}

[1] J. W. Cornejo. Prevalencia del trastorno por déficit de atención-hiperactividad en niños y adolescentes colombianos. Revista de neurologia, 2005; 40 (12): 716-722.

[2] C. Sánchez y A. Carrasco. Trastorno de déficit de atención e hiperactividad. Universidad de Valencia. 2015.

[3] M. T. Acosta. Trastorno deficitario de atenciónhiperactivad. Cap. 6. p. 119-120. Disponible en: http://www.acnweb.org/guia/g1c06i.pdf

Rev. Ingeniería, Matemáticas y Ciencias de la Información Vol. 3 / Núm. 6 / julio - diciembre de 2016; pág. 49-57 
[4] J. Vásquez. Guías Clínicas para la Atención de Trastornos Mentales. Guía Clínica para el trastorno de Déficit de Atención e Hiperactividad. México: Instituto Nacional de Psiquiatría Ramón de la Fuente Muñoz. 2010. p. 14.

[5] Trastorno por Déficit de Atención e Hiperactividad. Guía para Padres. En: Fundación de Neuropsicología Clínica. p. 8.

[6] E. M. García y A. Magaz. Psicólogos Clínicos y Educativos. Información sobre hiperactividad. Niños hiperactivos e inatentos. Grupo ALBOR-COHS. Fundación Calvida. 2007.

[7] M. Sánchez; J. Guerra y F. I. Revuelta. La relación de los videojuegos en el tratamiento del TDAH. Facultad de Formación del Profesorado. Universidad de Extremadura. 2011.

[8] P. Muñoz. Juego de ordenador para tratar la hiperactividad en niños: las nuevas tecnologías ayudan a niños con TDAH. Disponible en: Guiainfantil.com
[9] R. González. Acerca de tratamientos informáticos para el TDAH. En: Salud, 2015.

[10] VELSID. Videojuegos para tratar niños con TDAH. En: Péquela. 2013.

[11] B. Romo. MeMotiva, programa específico para el TDAH. Blog: EDUCADORES TDAH. 2014.

[12] MeMotiva Aplicación. En: REHASOFT. MeMotiva. Disponible en: http:/ / www.rehasoft.com/tdah/ memotiva-senior/

[13] B. Romo. PlayAttention, videojuego para el TDAH. Blog: EDUCADORES TDAH. 2014.

[14] Play Attention- Sheer Genius. NASCIA. Play Attention.

[15] A. Roa. Ejercicios y Juegos casero para niños hiperactivos. Blog: guiainfantil.com.

[16] J. Silver and E. Rosenbaum. Makey- Makey Classic. Blog: Makey- Makey. Disponible en: http://www.makeymakey.com/ 
